\title{
Corrigendum: Depletion of Arabidopsis ACYL-COA-BINDING PROTEIN3 Affects Fatty Acid Composition in the Phloem
}

\section{OPEN ACCESS}

Edited and reviewed by: Kent D. Chapman,

University of North Texas,

United States

*Correspondence: Mee-Len Chye mlchye@hku.hk

Specialty section: This article was submitted to Plant Physiology,

a section of the journal Frontiers in Plant Science

Received: 23 November 2020 Accepted: 26 January 2021 Published: 18 February 2021

Citation:

Hu T-H, Lung S-C, Ye Z-W and Chye M-L (2021) Corrigendum: Depletion of Arabidopsis ACYL-COA-BINDING PROTEIN3 Affects Fatty Acid Composition in the Phloem. Front. Plant Sci. 12:632503.

doi: 10.3389/fpls.2021.632503

\author{
Tai-Hua Hu, Shiu-Cheung Lung, Zi-Wei Ye and Mee-Len Chye* \\ School of Biological Sciences, The University of Hong Kong, Pokfulam, Hong Kong
}

Keywords: acyl-CoA esters, fatty acids, linolenic acid, jasmonate, oxylipins, wounding

\section{A Corrigendum on}

Depletion of Arabidopsis ACYL-COA-BINDING PROTEIN3 Affects Fatty Acid Composition in the Phloem

by Hu, T.-H., Lung, S.-C., Ye, Z.-W., and Chye, M.-L. (2018) Front. Plant Sci. 9:2. doi: $10.3389 / f p l s .2018 .00002$

In the original article, there was a mistake in Figure 3 as published. The representative images of the unwounded VC at $0 \mathrm{hpw}$ and the unwounded VC at $3 \mathrm{hpw}$ were duplicated. Figure $3 \mathrm{~B}$ has now been updated by replacing the two duplicated images with new representative images previously taken from the same VC batch in the experiment. The corrected Figure 3 appears below.

The authors apologize for this error and please note that the replaced images do not change the scientific conclusions of the article in any way. The original article has been updated.

Copyright $\odot 2021 \mathrm{Hu}$, Lung, Ye and Chye. This is an open-access article distributed under the terms of the Creative Commons Attribution License (CC BY). The use, distribution or reproduction in other forums is permitted, provided the original author(s) and the copyright owner(s) are credited and that the original publication in this journal is cited, in accordance with accepted academic practice. No use, distribution or reproduction is permitted which does not comply with these terms. 
A

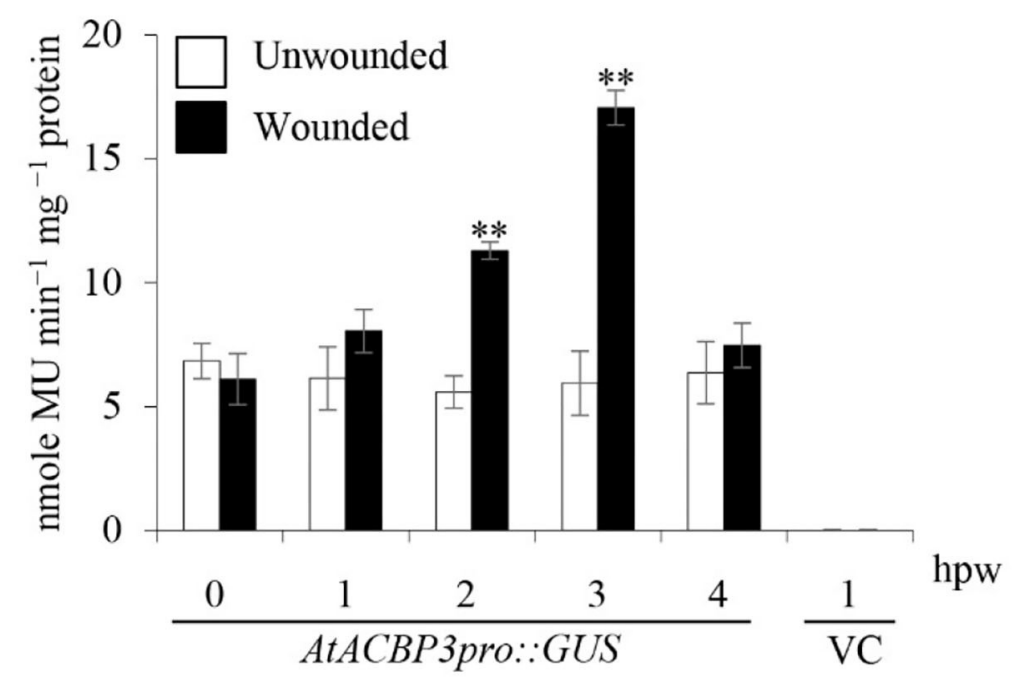

B

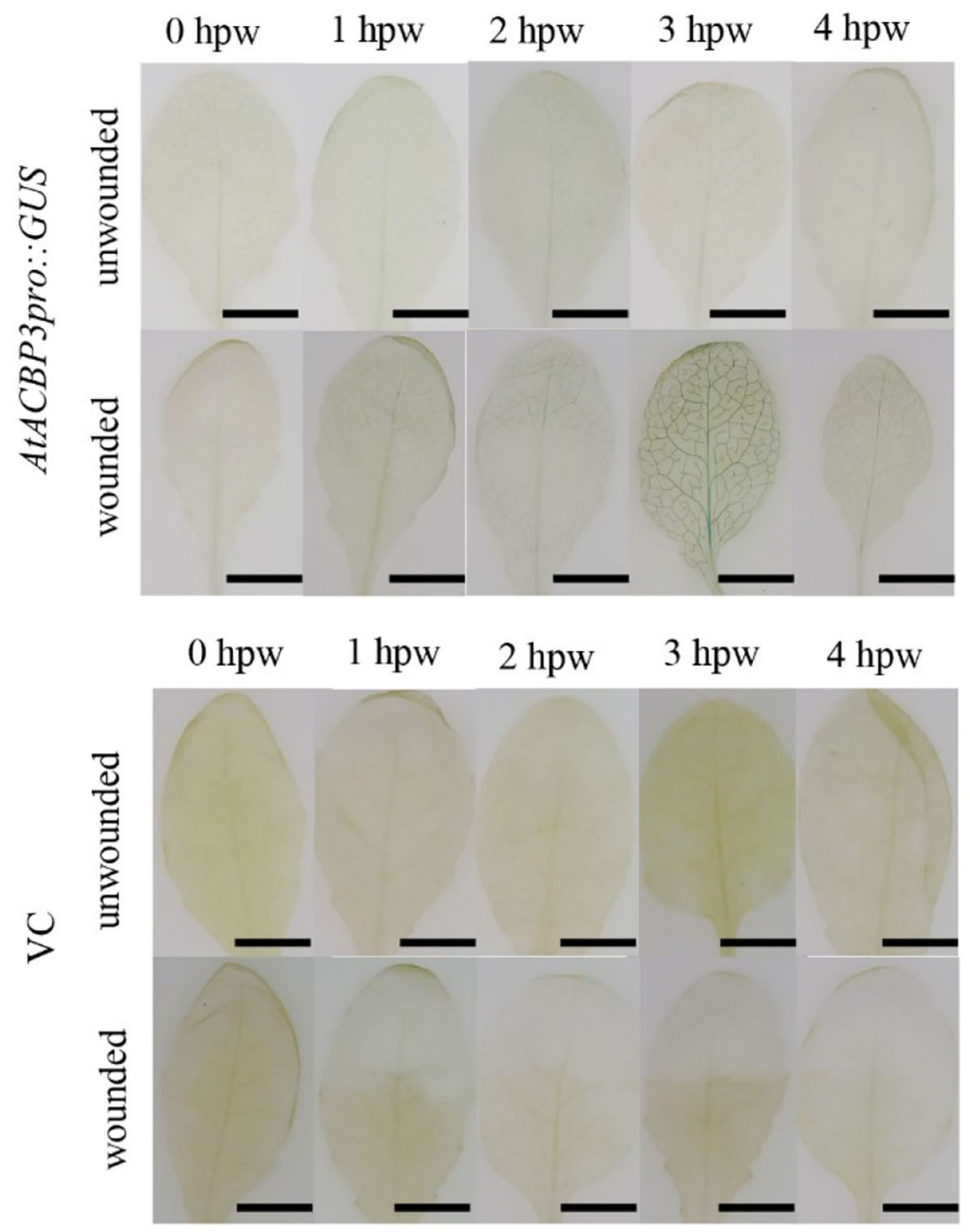

FIGURE 3 | GUS activity assays of transgenic Arabidopsis AtACBP3pro::GUS plants following wounding. (A) Four-week-old AtACBP3pro::GUS transgenic plants were wounded with a pair of forceps and harvested at 1, 2, 3, and $4 \mathrm{~h}$ post wounding (hpw) for quantitative GUS activity measurements. Vector

(pB|101.3)-transformed plants were analysed at $1 \mathrm{hpw}$ and served as a negative control, while unwounded AtACBP3pro::GUS plants were used as a baseline control. (Continued) 
FIGURE 3 | " "** indicates statistically significant difference $(P<0.01, n=3$ by Student's $t$-test) in comparison to unwounded samples collected at the same time point. Error bars represent standard deviations. (B) A representation of histochemical staining of wounded 4-week-old leaves from AtACBP3pro::GUS and vector (pBl101.3)-transformed (VC) Arabidopsis. These experiments were repeated twice with consistent results. Scale bars $=0.8 \mathrm{~cm}$. 